Monatsschr Kinderheilkd 2022 · 170:357-358 https://doi.org/10.1007/s00112-022-01445-4 Online publiziert: 7. März 2022

(C) The Author(s), under exclusive licence to Springer Medizin Verlag GmbH, ein Teil von Springer Nature 2022

\section{Erratum zu: Abstracts der 95. Wissenschaftlichen Tagung der Gesellschaft für Pädiatrische Onkologie und Hämatologie (GPOH)}

Vanessa Mettmann ${ }^{1,2} \cdot$ Claudia Blattmann $^{2} \cdot$ Matthias Kevric $^{2} \cdot$ Benjamin Sorg $^{2}$. Stefan Bielack ${ }^{2,3} \cdot$ Matthias Dürken $^{4}$. Stefanie Hecker-Nolting ${ }^{2}$

${ }^{1}$ Medizinische Fakultät Heidelberg, Universität Heidelberg, Heidelberg, Deutschland; ${ }^{2}$ Pädiatrie 5 (Onkologie, Hämatologie, Immunologie), Zentrum für Kinder-, Jugend- und Frauenmedizin, Stuttgart Cancer Center, Klinikum Stuttgart - Olgahospital, Stuttgart, Deutschland; ${ }^{3}$ Abteilung für Pädiatrische Hämatologie und Onkologie, Klinik für Kinder- und Jugendmedizin - Pädiatrische Hämatologie und Onkologie, Münster, Deutschland; ${ }^{4}$ Hämatologie und Onkologie, Klinik für Kinder- und Jugendmedizin, Universitätsmedizin Mannheim, Mannheim, Deutschland

\section{Erratum zu:}

Monatsschr Kinderheilkd 2021

https://doi.org/10.1007/s00112-021-

01353-z

Leider enthält der Abstract „Solitäre Lungenmetastasen als Erstrezidiv des hochmalignen Osteosarkoms bei 219 PatientInnen des COSS-Registers: Prognostische Faktoren, Rezidivtherapie und Überleben“ (S. 1239, Spalte 1) einen Tippfehler.

Dort steht im Abschnitt „Fragestellung":

Trotz kombinierter chirurgischer und systemischer Therapie tritt bei etwa 70\% der PatientInnen mit einem hochmalignen Osteosarkom ein Rezidiv auf.

Der Satz lautet korrekt:

Trotz kombinierter chirurgischer und systemischer Therapie tritt bei etwa $\mathbf{3 0} \%$ der PatientInnen mit einem hochmalignen Osteosarkom ein Rezidiv auf.

Wir bitten den Fehler zu entschuldigen.

\section{Korrespondenzadresse}

\section{Vanessa Mettmann}

Pädiatrie 5 (Onkologie, Hämatologie, Immunologie), Zentrum für Kinder-, Jugendund Frauenmedizin, Stuttgart Cancer Center, Klinikum Stuttgart - Olgahospital Stuttgart, Deutschland v.mettmann@klinikum-stuttgart.de
Die Online-Version des Originalartikels ist unter https://doi.org/10.1007/s00112-021-01353-Z zufinden. 
Hier steht eine Anzeige.

黑 Springer 